\title{
Uterine artery pseudoaneurysm after caesarean section: a rare cause of massive secondary postpartum haemorrhage
}

\author{
Kavita Agarwal, Rekha Bharati, Achla Batra, Aruna Batra \\ Correspondence: Dr. Kavita Agarwal, Assistant Professor, Department of Obstetrics and \\ Gynaecology, Safdarjung Hospital, New Delhi, India; Email : drku93@gmail.com
}

Distributed under Creative Commons Attribution-Share Alike 4.0 International.

\begin{abstract}
Postpartum haemorrhage (PPH) is one of the major causes of maternal morbidity and mortality. A 24 years old woman came to hospital with complain of profuse vaginal bleeding after 45 days of caesarean section. Ultrasound doppler report showed an anechoic cystic space occupying lesion in region of caesarean scar. It filled up colour and showed swirling motion and arterial doppler waveforms. So, lesion was diagnosed as uterine artery pseudoaneurysm and arterial embolisation was done for treatment. After that, since 7 months the patient is asymptomatic.
\end{abstract}

Keywords: Postpartum haemorrhage, pseudoaneurysm, arterial embolisation, caesarean section.

Postpartum haemorrhage (PPH) occurs in fewer than $5 \%$ of deliveries but is the major cause of maternal morbidity and mortality [1]. It can be primary, occurring within 24 hours resulting from uterine atony, traumatic, retained placental fragments and hypofibroginemia. Secondary or delayed PPH occurs after 24 hours to until 6 weeks of puerperium resulting from retained placental fragments, infection or from a rare cause such as pseudoaneurysm, arterio venous malformation or arterio-venous fistula. Pseudoaneurysm can have fatal complications due to rupture or haemorrhage. Haemorrhage in uterine cavity leads to delayed PPH and haemorrhage in peritoneal cavity results in pelvic collection. We report here a case of 24 year old female who presented to us as secondary postpartum haemorrhage following caesarean section. Diagnosis was established radiologically and uterine artery embolisation was successful in controlling the haemorrhage. The case is reported to help obstetricians in early recognition of this rare entity, thereby preventing life threatening complications.

\section{Case report}

Mrs X, 24 year old female, para 1, live 1, came to hospital with complaint of an episode of bleeding per vagina. She had caesarean section 45 days prior to admission in view of fetal distress at a neighbouring hospital. The case notes revealed extension at right angle of incision and average blood loss. She had an episode of bleeding per vagina on post-operative day 28 of caesarean section, went to a private hospital where pelvic ultrasound was done that was reported as

Received: $3^{\text {rd }}$ October 2016. Accepted: $16^{\text {th }}$ November 2016.

Agarwal K, Bharti R, Batra A, Batra A. Uterine artery pseudoaneurysm after caesarean section: a rare cause of massive secondary postpartum haemorrhage. The New Indian Journal of OBGYN. 2017; 3(2): 118-20 


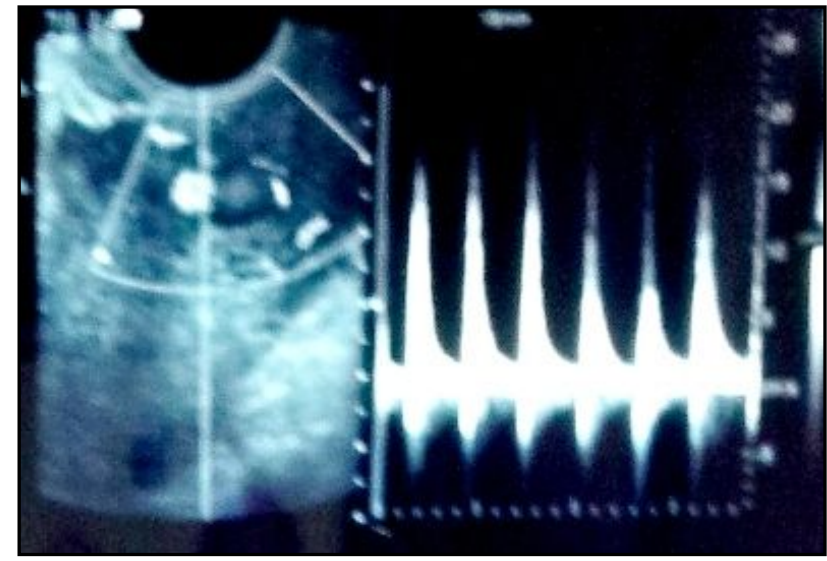

Figure 1: Ultrasound Doppler waveform

normal. There were no retained products of conception. Patient was managed conservatively on uterotonics and antibiotics. She again had a bout of bleeding per vaginum on post operative day 38 of caesarean section. Then she went to a private hospital where she was found to have severe anemia. Ultrasound was repeated which was found to be normal. She received 2 units of blood transfusion and iron sucrose injections.

The patient presented to our hospital with complaint of bleeding per vaginum since 1 day. Her physical and systemic examination was normal. Gynaecological examination revealed a normal size uterus with closed cervical os. Blood clots were seen at the cervical os. Pelvic ultrasound was done to rule out retained placental fragments. Ultrasound Doppler report showed an anechoic cystic space occupying lesion in region of caesarean scar, right of midline measuring $18 \times 16 \times 15 \mathrm{~mm}$. It filled up colour and showed swirling motion and arterial Doppler waveforms (Fig 1). It filled from its neck in right uterine artery at level of internal os and was reported as possibly pseudoaneurysm of right uterine artery. In consultation with interventional radiologist at another centre, uterine artery embolisation was done. After that, since 7 months the patient is asymptomatic.

\section{Discussion}

Uterine vascular abnormalities including pseudoaneurysm, acquired arterio venous malformation or arterio venous fistula occur following surgical trauma during caesarean section, myomectomy or uterine curettage [2]. Pseudoaneurysm differs from true aneurysm in that they are not surrounded by 3 layers of arterial wall. Most pseudoaneurysms result from vascular injuries, bleeding from the injured vessel forms haematoma in the surrounding tissues. If communication with the injured vessel remains patent, it leads to a perfused sac communicating with arterial lumen leading to formation of pseudoaneurysm. In present case, there was extension of the incision on right side which could had possibly led to formation of haematoma and hence pseudoaneurysm.

Pseudoaneurysm diagnosis is delayed because it is not considered in differential diagnosis of secondary PPH. Uterine artery pseudoaneurysm can easily be diagnosed by duplex Doppler sonography. The hallmark sign is to and fro waveform [2]. Although, various radiological techniques like ultrasound, MRI and Doppler have been used as tools for diagnosing arterio-venous malformations and pseudoaneurysm, pelvic angiography is considered the standard diagnostic method. In women presenting with delayed PPH without clinical evidence of endometritis or ultrasound evidence of retained products of conception, angiography should be performed at an early stage during management of such cases [3]. Delaying angiography could result in unnecessary morbidity and repeated blood transfusions as is reported in the present case.

The safe and effective treatment for controlling PPH due to uterine artery pseudoaneurysm is selective arterial embolisation of uterine artery preferably bilateral [1]. Recently, Sharma et al [3] reported results of study of uterine artery pseudoaneurysm managed successfully with uterine artery embolisation. Uterine artery embolisation done with selective catheterisation of the uterine arteries with Robert's uterine cathether and embolisation with polyvinyl alcohol $(250-300 \mu \mathrm{m})$ and fibred platinum coils. Several agents used for embolisation are gelatin sponge, polyvinyl alcohol particles, metallic coils. Gelatin sponge leads to temporary occlusion with potential for recanalisation 3 weeks later. Embolisation complications are 
uncommon. They include ischemia and abscess formation. Young Ho Choi [4] has reported 90\% overall success rates of uterine artery embolisation in patients with secondary postpartum haemorrhage.

Compared to arterial ligation and hysterectomy, arterial embolisation has high success rate, obviates need for general anaesthesia and major pelvic surgery, preserves fertility, has shorter hospital stay and avoids multiple transfusions.

\section{Conclusion}

Uterine artery pseudoaneurysm should always be kept in mind in patients with unexplained $\mathrm{PPH}$ especially after caesarean section. Pseudoaneurysm is easy to diagnose and treat. Delay in diagnosis can lead to prolonged morbidity, life threatening complications and multiple blood transfusions.

\section{Conflict of interest: None. Disclaimer: Nil.}

\section{References}

1.Yun SY, Lee DH, Cho KH, Le HM, Choi YH. Delayed postpartum haemorrhage resulting from uterine artery pseudoaneurysm rupture. Journal of Emergency

Medicine. 2012; 42: 11

2.Mou Y, Xu Y, Hu Y, Jiang T. Giant uterine artery pseudoaneurysm after a missed miscarriage termination in a caesarean scar pregnancy. BMC Women's Health. 2014;14:89

3.Sharma AM, Burbridge BE. Uterine artery

Pseudoaneurysm in the setting of delayed postpartum haemorrhage: successful treatment with emergency arterial embolisation. Case reports in Radiology. 2011; 2011: 373482

5.Choi YH, Baba Y, Ikeda S, So YH, Hayashi S, Nakajo M. Efficacy of uterine artery embolisation for patients with post partum haemorrhage. Open Journal of Radiology. 2013; 3: 51-5.

\section{Kavita Agarwal ${ }^{1}$, Rekha Bharati ${ }^{2}$, Achla Batra ${ }^{3}$, Aruna} Batra $^{4}$

${ }^{1,2}$ Assistant Professor, Department of Obstetrics and Gynaecology, Safdarjung Hospital, New Delhi, India; ${ }^{3}$ Associate Professor, Department of Obstetrics and Gynaecology, Safdarjung Hospital, New Delhi, India; ${ }^{4}$ Professor, Department of Obstetrics and Gynaecology, Safdarjung Hospital, New Delhi, India. 